\title{
An audit of the management of childhood- onset growth hormone deficiency during young adulthood in Scotland
}

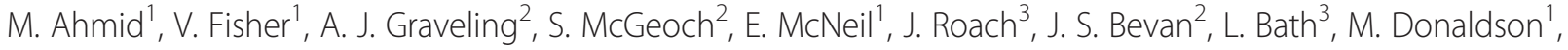 \\ G. Leese ${ }^{4}$, A. Mason' ${ }^{1}$ C. G. Perry ${ }^{5}$, N. N. Zammitt' ${ }^{6}$ S. F. Ahmed ${ }^{1}$ and M. G. Shaikh ${ }^{1 *}$
}

\begin{abstract}
Background: Adolescents with childhood onset growth hormone deficiency (CO-GHD) require re-evaluation of their growth hormone $(\mathrm{GH})$ axis on attainment of final height to determine eligibility for adult GH therapy (rhGH).

Aim: Retrospective multicentre review of management of young adults with CO-GHD in four paediatric centres in Scotland during transition.

Patients: Medical records of 130 eligible CO-GHD adolescents (78 males), who attained final height between 2005 and 2013 were reviewed. Median (range) age at initial diagnosis of CO-GHD was 10.7 years (0.1-16.4) with a stimulated $\mathrm{GH}$ peak of $2.3 \mu \mathrm{g} / \mathrm{l}$ (0.1-6.5). Median age at initiation of rhGH was 10.8 years (0.4-17.0).

Results: Of the 130 CO-GHD adolescents, 74/130(57 \%) had GH axis re-evaluation by stimulation tests /IGF-1 measurements. Of those, 61/74 (82 \%) remained GHD with 51/74 (69 \%) restarting adult rhGH. Predictors of persistent GHD included an organic hypothalamic-pituitary disorder and multiple pituitary hormone deficiencies (MPHD). Of the remaining 56/130 (43\%) patients who were not re-tested, 34/56 (61\%) were transferred to adult services on rhGH without biochemical retesting and 32/34 of these had MPHD. The proportion of adults who were offered rhGH without biochemical re-testing in the four centres ranged between 10 and $50 \%$ of their total cohort.

Conclusions: A substantial proportion of adults with CO-GHD remain GHD, particularly those with MPHD and most opt for treatment with rhGH. Despite clinical guidelines, there is significant variation in the management of CO-GHD in young adulthood across Scotland.
\end{abstract}

Keywords: Childhood onset growth hormone deficiency, Retesting, Re-evaluation, Transition, Adolescence

\section{Background}

The transition of care from childhood to adulthood for many chronic disorders requires a careful coordinated approach and this is particularly important in growth hormone deficiency (GHD). Traditionally, children with childhood onset GHD (CO-GHD) discontinue recombinant human GH therapy (rhGH) after attaining final height. However, adults with CO-GHD may have increased fat mass, decreased muscle mass and low bone mineral density, as well as reduced cardiac performance, altered lipid

\footnotetext{
* Correspondence: guftar.shaikh@nhs.net

'Developmental Endocrinology Research Group, Royal Hospital for Children, School of Medicine, University of Glasgow, 1345 Govan Road, Glasgow G51 4TF, UK

Full list of author information is available at the end of the article
}

status, reduced physical performance, impaired cognitive function and reduced well-being [1, 2]. Reports suggest that these adults may benefit from rhGH $[3,4]$. A number of studies have shown that a high proportion of CO-GHD patients remain $\mathrm{GH}$ deficient as adults especially those with multiple pituitary hormone deficiencies (MPHD) and/or structural abnormalities, whereas the majority of those with idiopathic or isolated GHD no longer have GHD in adulthood [5-7]. Therefore, after childhood treatment it is necessary to review GH status in order to assess appropriateness of adult rhGH replacement [8]. However, the extent of benefit from this therapy may be variable and the decision to reinstitute rhGH needs to be undertaken carefully. 
In this context, clinical practice guidelines have been issued on the subject of transition of care of young adults with CO-GHD [9-12]. However, the practicalities of these guidelines as well as the extent to which these guidelines have been implemented in clinical practice are unclear. The purpose of this multicentre study was to understand the variation that may exist in the management of young adults with CO-GHD after attainment of final height.

\section{Methods}

We reviewed databases from the four specialist endocrine centres in Scotland and identified young adults who had been diagnosed as having CO-GHD and who had been treated with $\mathrm{GH}$ during childhood and had subsequently reached final height between 2005 and 2013. Study entry criteria were: CO-GHD (low GH peak response on stimulation test $<6.6 \mu \mathrm{g} / \mathrm{l}), \mathrm{GH}$ treatment during childhood, attainment of final height between 2005 and 2013 (height velocity $<1 \mathrm{~cm} /$ year as defined in all centres), and evaluation of $\mathrm{GH}$ - axis by stimulation tests and/or IGF-1 levels after withdrawal of $\mathrm{GH}$ for at least one month. Exclusion criteria included: untreated CO-GHD, GH-treated patients with CO-GHD who have not yet attained final height. Baseline demographic data included: aetiology of CO-GHD, age at diagnosis of COGHD, duration of $\mathrm{GH}$ treatment, presence of multiple pituitary hormone deficiencies (MPHD), re-evaluation of $\mathrm{GH}$ axis, and whether adult $\mathrm{GH}$ treatment was recommenced or not. The persistent GHD after retesting for four centers was defined as cutoff $<5 \mu \mathrm{g} / \mathrm{L} \mathrm{GH}$ peak response for dynamic stimulation testes and/or low serum IGF-1 levels ( $<2$ SD for age and sex) [9]. IGF-1 level measurement for centres $\mathrm{A}, \mathrm{B}$, and $\mathrm{D}$ were done using IDS iSys and centre C measured IGF-1 levels by immunoassay on the Siemens Immulite. All IGF-1 levels were corrected for age and sex accordingly.

\section{Statistical analysis}

Data were analyzed using Minitab software (Version 16) with a significance level of $<0.05$ and are described as median, ranges and percentage. Additionally, the MannWhitney U-test was used for calculation of significance of differences between median values. Association with clinical factors was assessed by Spearman's rank coefficient and a positive predictive value (PPV) was calculated for the identified predictors of persistent GHD.

\section{Results}

\section{General characteristics}

A total of 142 patients were screened, 130 of whom met inclusion criteria. The 130 patients (78 male) comprised of: 70 from centre A, 32 from B, 18 from $C$ and 10 from D. Table 1 displays the aetiology of CO-GHD. Median age at diagnosis of CO-GHD was 10.7 years (0.1-16.4) with an initial stimulated $\mathrm{GH}$ peak of $2.3 \mu \mathrm{g} / \mathrm{l}(0.1-6.5)$, and basal IGF-1 was $74 \mu \mathrm{g} / \mathrm{l}(4.0-410.0)$. Median age at initiation of rhGH was 10.8 years (0.4-17.0). GH peak at diagnosis was lower in those with MPHD compared to IGHD $(1.9 \mu \mathrm{g} / \mathrm{l} \quad(<0.1-6.4)$ vs $3.0 \mu \mathrm{g} / \mathrm{l}(0.3-6.5)$ respectively: $p<0.01)$.

\section{Re-evaluation of $\mathrm{GH}$ axis}

A total of 74/130 (57\%) patients with CO-GHD (IGHD = $31(42 \%):$ MPHD $=43(58 \%))$ were biochemically retested at a median age of 18.2 years (14.5-21.3) (with one outlier patient who was retested at the age of 27.5 years), rhGH treatment was discontinued at the median age of 16.4 years (10.8-21.0). Biochemical retesting was performed after a median period of 0.5 years $(0.1-5.6)$ off rhGH $(21 / 74$ (28\%) were retested over period of ( $0.1-0.3$ years) and $34 /$ $74(46 \%)$ over a period of (0.4-5.7 years), with incomplete data on timing of re-testing in 20/74 (27\%). Median duration of childhood treatment was 5.3 years $(0.4-16.8)$. At retesting, the median $\mathrm{GH}$ peak was $1.6 \mu \mathrm{g} / \mathrm{l}(0.1-23.7)$ and IGF-1 was $88.0 \mu \mathrm{g} / \mathrm{l}$ (15.0-631.0). Of those retested, 61/74 (82\%) (32 males) remained GHD and were eligible for adult rhGH, with 51/61 (84\%) re-starting adult rhGH and $10 / 61$ (16 \%) declining therapy although it is possible that they may have restarted at a later stage. The remaining 13/74 (18 \%)(10 males) who were no longer GH deficient consisted of eight with idiopathic IGHD, two brothers with central hypothyroidism and normal pituitary MRI, one with an ectopic pituitary, one with hypogonadism and Coeliac disease and one with a history of cranial irradiation. Of the 56 of 130 (43\%) cases of CO-GHD who were not retested 34 (61 \%) were transferred from paediatric to adult services without biochemical retesting during transition, 12 (21\%) stopped treatment without biochemical reevaluation and 10 (18\%) were lost to follow up whilst on treatment (Fig. 1).

Dynamic function stimulation tests were performed in 40/74 (54 \%) patients who were retested, with 35/40 ( $88 \%$ ) of subjects having a low $\mathrm{GH}$ peak response $<5 \mu \mathrm{g} / \mathrm{l}$, with $27 / 35$ of them having severe GHD with a GH peak response $<3 \mu \mathrm{g} / \mathrm{l}$. Of the remaining 34/74 (46\%) patients who were retested, IGF-1 levels alone were available and low enough to confirm GHD ( $\leq-2$ SD for age and gender) in 19/34 (56 \%) of which 15 had MPHD and 4 had IGHD (organic causes and abnormal pituitary MRI). Two patients (MPHD) had IGF-1 levels within normal range on initial retesting ( $>2 \mathrm{SD}$ for age and gender), but were confirmed to have GHD following GH stimulation tests.

\section{Reconfirmation of GHD and initiation of adult GH replacement therapy}

Of the 130 with CO-GHD, 34 (26\%) patients continued adult rhGH without temporary cessation of therapy or 
Table 1 The categories of patients with CO-GHD according to aetiology and centres distribution is shown as (A, B, C, D)

\begin{tabular}{|c|c|c|c|}
\hline & Total number & IGHD & MPHD* \\
\hline & & 48/130 (37\%) & $82 / 130(63 \%)$ \\
\hline Congenital $n(\%)(A, B, C, D)$ & $38 / 130(29 \%)$ & $12(8,1,2,1)$ & $26(14,3,4,5)$ \\
\hline -Pituitary axial structural abnormalities $(A, B, C, D)$ & 24 & $9(6,1,1,1)$ & $15(6,3,1,5)$ \\
\hline -Midline axial structure defects (SOD) $(A, B, C, D)$ & 14 & $3(2,0,1,0)$ & $11(8,0,3,0)$ \\
\hline Oncology/cranial irradiation $n(\%)(A, B, C, D)$ & $51 / 130(40 \%)$ & $8(5,3,0,0)$ & $43(18,19,4,2)$ \\
\hline - Craniopharyngioma $(A, B, C, D)$ & 15 & - & $15(6,7,1,1)$ \\
\hline - Hematologic malignancies $(A, B, C, D)$ & 12 & $4(4,0,0,0)$ & $8(6,0,1,1)$ \\
\hline - Medulloblastoma $(A, B, C, D)$ & 6 & $1(0,1,0,0)$ & $5(1,4,0,0)$ \\
\hline - Other CNS tumors $(A, B, C, D)$ & 18 & $3(1,2,0,0)$ & $15(5,8,2,0)$ \\
\hline Idiopathic $n$ (\%) $(\mathrm{A}, \mathrm{B}, \mathrm{C}, \mathrm{D})$ & 15/130 (11\%) & $13(7,1,5,0)$ & $2(1,1,0,0)$ \\
\hline Others $^{\mathrm{b}} n(\%)(\mathrm{A}, \mathrm{B}, \mathrm{C}, \mathrm{D})$ & 26/130 (20\%) & $15(12,1,1,1)$ & $11(5,3,2,1)$ \\
\hline -Crohn's disease $(A, B, C, D)$ & 4 & $4(3,0,0,1)$ & - \\
\hline -Coeliac disease $(A, B, C, D)$ & 2 & - & $2(0,1,1,0)$ \\
\hline -Haematological diseases ${ }^{c}(A, B, C, D)$ & 2 & $1(1,0,0,0)$ & $1(1,0,0,0)$ \\
\hline -Other diseases ${ }^{d}(A, B, C, D)$ & 11 & $8(6,1,1,0)$ & $3(0,2,1,0)$ \\
\hline -Syndromes ${ }^{\mathrm{e}}(\mathrm{A}, \mathrm{B}, \mathrm{C}, \mathrm{D})$ & 6 & $2(2,0,0,0)$ & $4(3,0,0,1)$ \\
\hline -Acquired brain injury $(A, B, C, D)$ & 1 & - & $1(1,0,0,0)$ \\
\hline
\end{tabular}

Data are presented as the numbers of patients and percentages are given in parentheses

*33/82 patients with one additional pituitary hormone deficiency, 17/82 with two additional deficiencies, 19/82 with three and 13/82 with four additional deficiencies 'panhypopituitarism'

IGHD, isolated growth hormone deficiency; MPHD, multiple-pituitary hormone deficiencies; SOD, Septo-optic dysplasia

${ }^{a}$ Normal pituitary MRI, GHD is not associated with other conditions

${ }^{\mathrm{b}}$ Normal pituitary MRI (or no MRI report), but GHD is associated with other conditions

'(Thalassemia, X-linked Sideroblastic Anaemia)

${ }^{\mathrm{d}}$ (Microephaly with learning disability, history of intrauterine growth retardation, gastrochisis with history of small for gestational age, Asthma, joint hypermobility syndrome, pesudohypoparathyrodism)

${ }^{e}$ (Charge syndrome, Noonan syndrome, Kallman Syndrome, trisomy 22, Klinefelter's syndrome, Turner's syndrome with GHD)

formal retesting. Of these 34, nine had structural abnormalities on MRI, 22 were related to late effects of cancer therapy and three had unexplained GHD. Of those 34, 31 (91\%) had MPHD (17/32 of them had three or more additional pituitary hormone deficiencies (PHDs)); and 3/34 (9 \%) had IGHD (two with pituitary structural abnormalities on MRI and one with tumour related GHD).
These patients were advised to continue rhGH until their mid-20s.

For patients who were re-tested, GH cut offs for considering rhGH varied between centres. Not all patients found to have persistent GHD restarted adult $\mathrm{GH}$ therapy despite low peak GH levels at re-testing. There were four patients who were found not to have severe GHD with GH peaks

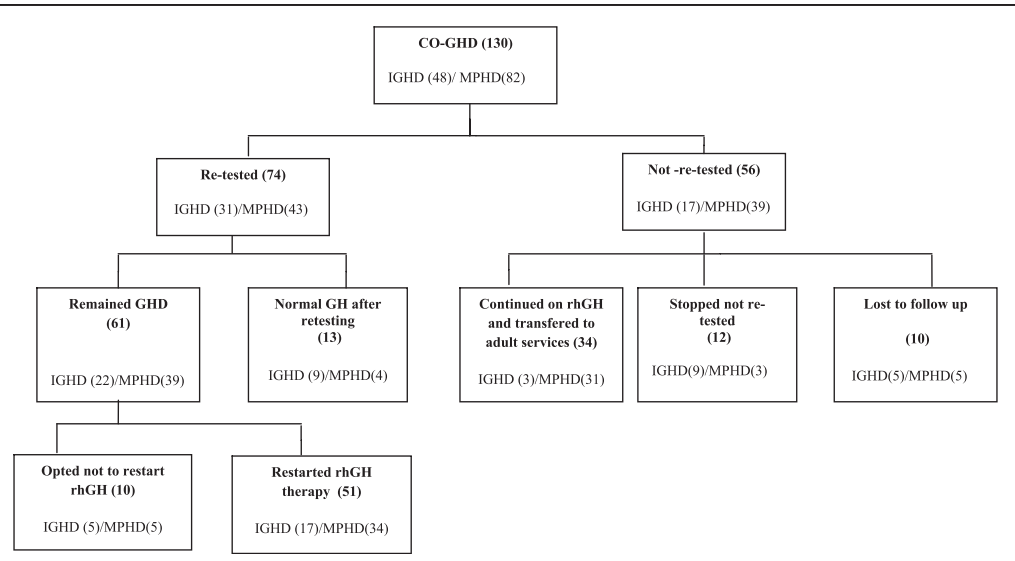

Fig. 1 Study Cohort Flow Chart. (n); number of patients, CO-GHD; childhood onset growth hormone deficiency, IGHD; isolated growth hormone deficiency, MPHD; multiple-pituitary hormone deficiencies, GH; growth hormone therapy 
4-5 $\mu \mathrm{g} / \mathrm{l}$ (three patients from centre B, one from centre A) and were not offered rhGH as they did not meet adult criteria for replacement. However, among those who were offered rhGH after retesting, one patient with IGHD (centre C) had a GH peak >5 $\mu \mathrm{g} / \mathrm{l}(5.5 \mu \mathrm{g} / \mathrm{l})$.

\section{Variation in the management between centres}

There were substantial variations in the management of CO-GHD between Scottish centres. Re-testing with stimulation testes and/or IGF-1 levels was found to be the highest in centre A (68\%), while centre C had the lowest percentage of retested patients ( $28 \%$ ), although this did include all IGHD patients from centre C. Centre B did not retest those with a high likelihood of permanent GHD (especially those with three or more additional PHDs) and had the highest percentage of adults on rhGH without biochemical re-evaluation (Table 2). A total of 32/130 patients in the cohort had three or more additional PHDs (Table 3). Of these 32, 14 (44\%) were retested using their IGF-1 levels alone and all were confirmed to have adult GHD, 17 (53 \%) continued on rhGH without biochemical retesting and one was lost to follow up whilst on treatment.

\section{Predictors of persistent GHD on re-evaluation}

Patients with persistent GHD were diagnosed at an earlier age $((8.4$ years $(0.3-16.0)$ vs 11.6 years $(7.1-15.5))$ and reached final height with re-evaluation of their $\mathrm{GH}$ axis in earlier adolescence ((17.9 years $(14.2-21.2)$ vs 19.3 years (17.3-21.3)) than those who were no longer GH deficient on retesting. No significant differences in the other parameters between persistent GHD and nonpersistent GHD were identified at time of diagnosis or re-evaluation. In this population the peak $\mathrm{GH}$ level on retesting was positively related with the $\mathrm{GH}$ peak level at childhood $(r=0.4, P=0.02)$. The number of additional PHDs was a predictor of a low peak GH on retesting as all patients with two or more additional PHDs had a lower GH response $(<5 \mu \mathrm{g} / \mathrm{l})$ at reassessment with a PPV (93\%). The presence of hypothalamic-pituitary structural abnormalities has a high PPV (96\%) of persistent
GHD, as of the 25 who were retested, 24 were reconfirmed with persistent GHD. Similarly, CO-GHD with a history of cranial irradiation predicted persistent GHD in adulthood (96\%).

\section{Discussion}

Our data confirm that a high proportion (82\%) of the retested patients with $\mathrm{CO}-\mathrm{GHD}$ continue to have GHD as adults. The majority ( $80 \%$ ) of those who remain $\mathrm{GH}$ deficient opted to resume adult GH treatment, however it is unknown whether they complied with therapy and for how long they continued with the treatment. It may also be the case that those adults who were GHD initially declined to restart rhGH during transition, but later reconsidered GH therapy. Factors influencing this decision would be an important area for future studies.

Previous published studies have reported variable estimates of persistent GHD in adulthood ranging from $12.5-90 \%[13,14]$ but the high incidence of ongoing GHD in adulthood in our cohort may be attributed to the large proportion of patients with organic causes for their GHD. Some of our subjects with MPHD who had no structural abnormalities on MRI continued to have GHD, raising the possibility of an underlying genetic disorder. Similarly, the majority of idiopathic IGHD who were re-evaluated were $\mathrm{GH}$ deficient which may also indicate an underlying genetic predisposition. These findings suggest the importance of follow up and regular assessment of pituitary function in those with a low probability of persistent GHD, as they may develop other pituitary hormone deficiencies as previously demonstrated $[15,16]$. On the other hand, some patients who would be considered as having a moderate to high probability of persistent GHD (IGHD with structural abnormalities, patients with MPHD or those with a history of cranial irradiation) were no longer GH deficient. These findings demonstrate the limitations in using "at risk" groups to determine who require re-evaluation of their $\mathrm{GH}$ axis and those who do not.

Our data confirm that while there are no unequivocal auxological or clinical signs that predict the transiency

Table 2 Management of patients with CO-GHD according to each Scottish centre

\begin{tabular}{|c|c|c|c|c|c|}
\hline & All centres & A & B & C & $\mathrm{D}$ \\
\hline Total number of patients $(n)$ & 130 & 70 & 32 & 18 & 10 \\
\hline Total number of patients re-tested $n(\%)$ & $74 / 130(57)$ & $48 / 70(69)$ & $16 / 32(50)$ & $5 / 18(28)$ & $5 / 10(50)$ \\
\hline Persistent GHD $n(\%)$ & $61 / 74(82)$ & $43 / 48(90)$ & $12 / 16(75)$ & $1 / 5(20)$ & $5 / 5(100)$ \\
\hline Those with persistent GHD who restarted rhGH $n(\%)$ & $51 / 61(83)$ & $35 / 43(81)$ & $11 / 12(92)$ & $1 / 1(100)$ & $4 / 5(80)$ \\
\hline Total number of patients not-retested $n(\%)$ & $56 / 130(43)$ & $22 / 70(31)$ & $16 / 32(50)$ & $13 / 18(72)$ & $5 / 10(50)$ \\
\hline Continued adult rhGH therapy without re-testing $n(\%)$ & $34 / 56(61)$ & $7 / 22(32)$ & $16 / 16(100)$ & $7 / 13(54)$ & $4 / 5(80)$ \\
\hline Lost to follow up whilst on treatment $n(\%)$ & 10/56 (18) & $9 / 22(41)$ & 0 & 0 & $1 / 5(20)$ \\
\hline Stopped $\mathrm{GH}$, no re-testing required $n(\%)$ & $12 / 56(21)$ & $6 / 22(27)$ & 0 & $6 / 13(46)$ & 0 \\
\hline
\end{tabular}

Data are presented as the numbers of patients and percentages are given in parentheses 
Table 3 Variation in the management of patients with CO-GHD between the four Scottish centres according to GHD categories

\begin{tabular}{|c|c|c|c|c|c|c|c|c|}
\hline \multirow[t]{2}{*}{ Centres } & \multicolumn{2}{|l|}{ A } & \multicolumn{2}{|l|}{ B } & \multicolumn{2}{|l|}{ C } & \multicolumn{2}{|l|}{$\mathrm{D}$} \\
\hline & IGHD & MPHD & IGHD & $\mathrm{MPHD}$ & IGHD & $\mathrm{MPHD}$ & IGHD & MPHD \\
\hline Total CO-GHD $n=130[32]$ & 32 & $38[16]$ & 6 & $26[10]$ & 8 & $10[3]$ & 2 & $8[3]$ \\
\hline Retested $n=74[14]$ & 21 & $27[13]$ & 4 & $12[0]$ & 4 & $1[0]$ & 2 & $3[1]$ \\
\hline With structural abnormalities ${ }^{a}$ & 8 & $13[5]$ & - & 2 & 1 & - & - & - \\
\hline Tumour related ${ }^{\mathrm{b}}$ & 3 & $13[8]$ & 2 & 7 & - & 1 & - & 1 \\
\hline Idiopathic/unexplained ${ }^{c}$ & 10 & 1 & 2 & 3 & 3 & - & 2 & $2[1]$ \\
\hline Re-confirmed GHD & 16 & $27[13]$ & 3 & $9[0]$ & 1 & 0 & 2 & $3[1]$ \\
\hline Not retested (but on adult rhGH) $n=34$ [17] & 0 & $7[2]$ & 2 & $14[10]$ & 1 & $6[3]$ & - & $4[2]$ \\
\hline With structural abnormalities ${ }^{a}$ & - & - & 2 & 1 & 1 & $3[3]$ & - & 1 \\
\hline Tumour related ${ }^{\mathrm{b}}$ & - & $5[2]$ & - & $12[10]$ & - & 3 & - & $1[1]$ \\
\hline Idiopathic/unexplained ${ }^{c}$ & - & 2 & - & 1 & - & - & - & $2[1]$ \\
\hline
\end{tabular}

Data are presented as number of patients with CO-GHD and [number of patients who have three and more additional pituitary hormones deficiencies

${ }^{a} \mathrm{MRI}$ imaging reported hypothalamic-pituitary axial structural abnormalities

${ }^{\mathrm{b}}$ Cranial irradiation

${ }^{\mathrm{C}}$ Normal pituitary MRI/Congenital GHD unexplained (no MRI report)/and/or associated with chronic disease

or the persistence of GHD, a history of organic disease, the presence of two or more additional PHDs [17, 18], presence of hypothalamic-pituitary structural abnormalities and tumour related organic GHD are strong indicators of persistence GHD [19-22].

In terms of timing of retesting, the current guidelines suggest that a period from one to three months off rhGH is sufficient for retesting [9]. Our data show a variable interval between stopping treatment and reassessment, with only $28 \%$ of patients off rhGH for less than 3 months. It is not clear for those who were off rhGH for longer duration before reassessment whether their stopping rhGH was for reasons other than re-testing. However, this prolonged period off rhGH may be associated with detrimental effects on somatic bone and body composition development during transition [23, 24], with recommendations for prompt resumption of rhGH in individuals with clinical evidence of persistent GHD [25]. Furthermore, a longer interval off rhGH may increase the risk of being lost to follow up in these already vulnerable patients and continued follow up around this time is essential [26]. We recommend that in patients who are under the care of paediatric services, the evaluation of GHD in transition should be undertaken by the paediatric clinic, ideally in the context of a joint transition service to improve the follow up and smooth transfer of adolescents with chronic endocrine conditions to the adult services as previously suggested [27].

The principle of offering rhGH during transition for those who have ongoing severe deficiency was variable between centres as the cut-offs chosen are variable, though the majority were in keeping with the guidance suggesting a GH peak $<5 \mu \mathrm{g} / \mathrm{l}$ constituting severe GHD in transition $[9,10]$. Few patients in our cohort declined restarting adult $\mathrm{rhGH}$, they may be asymptomatic and therefore reluctant to restart rhGH therapy. Approximately one third of our subjects were considered to be very likely to have permanent GHD and therefore continued rhGH uninterrupted, apart from adjustment to an adult $\mathrm{GH}$ dose. This is in line with current guidelines which recommend that patients with severe congenital or acquired panhypopituitarism with three or more pituitary hormone deficiencies or identified genetic mutations may not require re-evaluation of their $\mathrm{GH}$ status; otherwise all patients with CO-GHD require biochemical re-evaluation and reconfirmation of GHD during transition before reinstituting adult GH replacement therapy $[9,12]$. However, of the 34 patients who continued adult rhGH without formal retesting, nine had structural abnormalities on MRI probably were at high risk of ongoing GHD, but three had unexplained GHD and probably should have been retested. Furthermore, some centres still retested those with a high likelihood of permanent GHD, by checking their IGF1 levels, although all were reconfirmed GHD and resumed rhGH. On these grounds, it seems that no clear consensus has been reached as to whether or not to withdraw treatment and retest those at high risk of permanent GHD. It is also unclear whether those who continued rhGH without biochemical re-testing were reevaluated at a later stage. For those who restarted rhGH, according to the current guidelines, at completion of somatic growth (approximately 25-30 years old) further re-evaluation should be undertaken with the offer of adult GH replacement therapy and monitoring in accordance with National Institute for Health and Care Excellence guidance (NICE) (TA 64 August 2003).

\section{Conclusion}

In conclusion, this study not only provided a snapshot of the differences in management of CO-GHD during 
transition across Scotland, but it has also enabled us to identify areas of uncertainty despite there being clinical practice recommendations. Our data showed a substantial proportion of patients with CO-GHD remain GH deficient and most opt for rhGH as adults, although not all patients may require re-evaluation of their $\mathrm{GH}$ axis. This study also raises concerns about follow up of those who no longer have GHD and patients with GHD who opted not to resume adult rhGH. The optimal management of adolescents with CO-GHD requires continuous follow up during transition and effective communication between paediatric and adult services.

\begin{abstract}
Abbreviations
CO-GHD: childhood onset growth hormone deficiency; GH: growth hormone; GHD: growth hormone deficiency; IGF-1: insulin-like growth factor 1; IGHD: isolated growth hormone deficiency; MPHD: multiple pituitary hormone deficiencies; MRI: magnetic resonance imaging; PPV: positive predictive value; SDS: standard deviation score.
\end{abstract}

\section{Competing interests}

The authors declare that they have no competing interests.

\section{Authors' contributions}

MA and MGS designed, performed data collection, performed the main part of statistical analysis, and wrote the initial draft of the manuscript. SFA conceived of the study and participated in its design and coordination as well as drafting the manuscript. AJG, SM, GL, JSB contributed in data collection and interpretations. All authors made substantial contributions read, edited and approved the final manuscript.

\section{Acknowledgements}

MA is currently supported by the Government of Libya.

\section{Author details}

'Developmental Endocrinology Research Group, Royal Hospital for Children, School of Medicine, University of Glasgow, 1345 Govan Road, Glasgow G51 4TF, UK. ${ }^{2}$ JJR Macleod Centre for Diabetes, Endocrinology \& Metabolism, Aberdeen Royal Infirmary, Aberdeen, UK. ${ }^{3}$ Department of Endocrinology, Royal Hospital for Sick Children, Edinburgh, UK. ${ }^{4}$ Ninewells Hospital and Medical School in Dundee, Dundee, UK. ${ }^{5}$ Department of Endocrinology, Queen Elizabeth University Hospitals, Glasgow, UK. ${ }^{6}$ Royal Infirmary of Edinburgh, Edinburgh, UK.

Received: 29 October 2015 Accepted: 27 January 2016 Published online: 16 March 2016

\section{References}

1. deBoer $\mathrm{H}$, vanderVeen EA. Why retest young adults with childhood-onset growth hormone deficiency? J Clin Endocrinol Metab. 1997;82(7):2032-6.

2. Moller N, Jorgensen JO. Effects of growth hormone on glucose, lipid, and protein metabolism in human subjects. Endocr Rev. 2009;30(2):152-77.

3. Carroll PV, Christ ER, Bengtsson BA, Carlsson L, Christiansen JS, Clemmons D, et al. Growth hormone deficiency in adulthood and the effects of growth hormone replacement: a review. Growth Hormone Research Society Scientific Committee. J Clin Endocrinol Metab. 1998;83(2):382-95.

4. Clayton P, Gleeson H, Monson J, Popovic V, Shalet SM, Christiansen JS. Growth hormone replacement throughout life: insights into age-related responses to treatment. Growth Horm IGF Res. 2007;17(5):369-82.

5. Geffner ME. Growth hormone replacement therapy: transition from adolescence to adulthood. J Clin Res Pediatr Endocrinol. 2009;1(5):205-8.

6. Tauber M, Moulin P, Pienkowski C, Jouret B, Rochiccioli P. Growth hormone $(\mathrm{GH})$ retesting and auxological data in $131 \mathrm{GH}$-deficient patients after completion of treatment. J Clin Endocrinol Metab. 1997;82(2):352-6.

7. Loche S, Bizzarri C, Maghnie M, Faedda A, Tzialla C, Autelli M, et al. Results of early reevaluation of growth hormone secretion in short children with apparent growth hormone deficiency. J Pediatr. 2002;140(4):445-9.
8. Koltowska-Haggstrom M, Geffner ME, Jonsson P, Monson JP, Abs R, Hana V, et al. Discontinuation of growth hormone (GH) treatment during the transition phase is an important factor determining the phenotype of young adults with nonidiopathic childhood-onset GH deficiency. J Clin Endocrinol Metab. 2010;95(6):2646-54

9. Clayton PE, Cuneo RC, Juul A, Monson JP, Shalet SM, Tauber M, et al. Consensus statement on the management of the $\mathrm{GH}$-treated adolescent in the transition to adult care. Eur J Endocrinol. 2005;152(2):165-70.

10. Radovick S, Divall S. Approach to the growth hormone-deficient child during transition to adulthood. J Clin Endocrinol Metab. 2007;92(4):1195-200.

11. Ho KK. Consensus guidelines for the diagnosis and treatment of adults with GH deficiency II: a statement of the GH Research Society in association with the European Society for Pediatric Endocrinology, Lawson Wilkins Society, European Society of Endocrinology, Japan Endocrine Society, and Endocrine Society of Australia. Eur J Endocrinol. 2007:157(6):695-700.

12. Cook DM, Rose SR. A review of guidelines for use of growth hormone in pediatric and transition patients. Pituitary. 2012:15(3):301-10.

13. Maghnie M, Strigazzi C, Tinelli C, Autelli M, Cisternino M, Loche S, et al. Growth hormone (GH) deficiency (GHD) of childhood onset: reassessment of $\mathrm{GH}$ status and evaluation of the predictive criteria for permanent GHD in young adults. J Clin Endocrinol Metab. 1999;84(4):1324-8.

14. Attanasio AF, Howell S, Bates PC, Blum WF, Frewer P, Quigley C, et al. Confirmation of severe $\mathrm{GH}$ deficiency after final height in patients diagnosed as GH deficient during childhood. Clin Endocrinol (Oxf). 2002;56(4):503-7.

15. Lange M, Feldt-Rasmussen U, Svendsen OL, Kastrup KW, Juul A, Muller J. High risk of adrenal insufficiency in adults previously treated for idiopathic childhood onset growth hormone deficiency. J Clin Endocrinol Metab. 2003;88(12):5784-9.

16. Blum WF, Deal C, Zimmermann AG, Shavrikova EP, Child CJ, Quigley CA, et al. Development of additional pituitary hormone deficiencies in pediatric patients originally diagnosed with idiopathic isolated $\mathrm{GH}$ deficiency. Eur J Endocrinol. 2014;170(1):13-21.

17. Juul A, Holm K, Kastrup KW, Pedersen SA, Michaelsen KF, Scheike T, et al. Free insulin-like growth factor I serum levels in 1430 healthy children and adults, and its diagnostic value in patients suspected of growth hormone deficiency. J Clin Endocrinol Metab. 1997;82(8):2497-502.

18. Hartman ML, Crowe BJ, Biller BM, Ho KK, Clemmons DR, Chipman JJ, et al. Which patients do not require a GH stimulation test for the diagnosis of adult GH deficiency? J Clin Endocrinol Metab. 2002;87(2):477-85.

19. Tillmann V, Tang WW, Price DA, Hughes DG, Wright NB, Clayton PE. Magnetic resonance imaging of the hypothalamic-pituitary axis in the diagnosis of growth hormone deficiency. J Pediatr Endocrinol Metab. 2000;13(9):1577-83.

20. Kalina MA, Kalina-Faska B, Gruszczynska K, Baron J, Malecka-Tendera E. Usefulness of magnetic resonance findings of the hypothalamic-pituitary region in the management of short children with growth hormone deficiency: evidence from a longitudinal study. Childs Nerv Syst. 2012;28(1):121-7.

21. Bonfig W, Bechtold S, Bachmann S, Putzker S, Fuchs O, Pagel P, et al. Reassessment of the optimal growth hormone cut-off level in insulin tolerance testing for growth hormone secretion in patients with childhood-onset growth hormone deficiency during transition to adulthood. J Pediatr Endocrinol Metab. 2008:21(11):1049-56.

22. Quigley CA, Zagar AJ, Liu CC, Brown DM, Huseman C, Levitsky L, et al. United States multicenter study of factors predicting the persistence of $\mathrm{GH}$ deficiency during the transition period between childhood and adulthood. Int J Pediatr Endocrinol. 2013;2013(1):6. doi:10.1186/1687-9856-2013-6.

23. Carroll PV, Drake WM, Maher KT, Metcalfe K, Shaw NJ, Dunger DB, et al. Comparison of continuation or cessation of growth hormone (GH) therapy on body composition and metabolic status in adolescents with severe $\mathrm{GH}$ deficiency at completion of linear growth. J Clin Endocrinol Metab. 2004:89(8):3890-5.

24. Tritos NA, Hamrahian AH, King D, Greenspan SL, Cook DM, Jonsson PJ, et al. A longer interval without $\mathrm{GH}$ replacement and female gender are associated with lower bone mineral density in adults with childhood-onset GH deficiency: a KIMS database analysis. Eur J Endocrinol. 2012;167(3):343-51.

25. Nguyen VT, Misra M. Transitioning of children with GH deficiency to adult dosing: changes in body composition. Pituitary. 2009;12(2):125-35.

26. Courtillot C, Baudoin R, Du Souich T, Saatdjian L, Tejedor I, Pinto G, et al. Monocentric study of 112 consecutive patients with childhood onset GH deficiency around and after transition. Eur J Endocrinol. 2013;169(5):587-96.

27. Downing J, Gleeson HK, Clayton PE, Davis JR, Wales JK, Callery P. Transition in endocrinology: the challenge of maintaining continuity. Clin Endocrinol (Oxf). 2013;78(1):29-35. 